\title{
QUIZIZZ Y SU APLICACIÓN EN EL APRENDIZAJE DE LOS ESTUDIANTES DE LA CARRERA PROFESIONAL DE IDIOMA EXTRANJERO
}

\author{
QUIZIZZ AND ITS APPLICATION IN LEARNING OF THE STUDENTS \\ OF THE FOREIGN LANGUAGE PROFESSIONAL CAREER
}

\author{
Hillary Eddy Robles Gonzales ${ }^{1 *}$, Ruth Xiomara Salamanca Chaparro ${ }^{1}$, Kevin Mario Laura De La \\ Cruz $^{1}$ \\ hroblesg@unjbg.edu.pe; rsalamancac@unjbg.edu.pe; klaurac@unjbg.edu.pe \\ ${ }^{1}$ Universidad Nacional Jorge Basadre Grohmann, Tacna, Perú
}

*Correspondencia: Hillary Eddy Robles Gonzales. Email: hroblesg@unjbg.edu.pe

Recibido: 14.08.21 | Aprobado: 30.08.21

\section{RESUMEN}

La inserción de las diversas plataformas educativas ha facilitado el proceso virtual de la enseñanza. Una de las herramientas más empleadas es Quizizz en la retroalimentación y puede generar diferentes estilos de aprendizaje. La presente investigación tiene como objetivo describir la percepción de Quizizz como parte del conectivismo en la educación superior de la Universidad Nacional Jorge Basadre Grohmann en Tacna-Perú en el año académico 2021. El tipo de investigación es descriptiva, mientras que el método empleado es inductivo. Para su ejecución se trabajó mediante un formulario virtual conformado por 13 preguntas, considerando 168 estudiantes de la especialidad Idioma Extranjero de la Facultad de Educación como muestra del presente estudio. Los resultados demuestran que la plataforma Quizizz incentiva al estudiante a conectar con su aprendizaje de manera positiva. Como conclusión, los estudiantes consideran relevante a las herramientas tecnológicas para lograr un aprendizaje óptimo, puesto que la tecnología ya forma parte de su proceso de aprendizaje, y con la conducción del docente pueden lograr una clase dinámica, donde participen activamente.

Palabras clave: Aprendizaje, Conectivismo, Educación Superior, Herramientas digitales.

\begin{abstract}
The insertion of the various educational platforms has facilitated the virtual teaching process. One of the most used tools is Quizizz in feedback and it can generate different learning styles. The present research aims to describe the perception of Quizizz as part of connectivism in higher education at the Jorge Basadre Grohmann National University in Tacna-Peru in the academic year 2021. The type of research is descriptive, while the method used is inductive. For its execution, a virtual form made up of 13 questions was used, considering 168 students of the Foreign Language specialty of the Faculty of Education as a sample of this study. The results show that the Quizizz platform encourages students to connect with their learning in a positive way. In conclusion, students consider technological tools relevant to achieve optimal learning, since technology is already part of their learning process, and with the teacher's guidance, they can achieve a dynamic class, where they actively participate.
\end{abstract}

Keywords: Learning, Connectivism, Higher Education, Digital tools. 


\section{INTRODUCCIÓN}

En concordancia con Downes (2007) el conectivismo es un aprendizaje inmerso en la era digital que se expande mediante una red de conexiones, que destacan por la capacidad de generar nuevos estilos de aprendizaje en red. Así mismo se caracteriza como el progreso frente a cómo el estudiante se desenvuelve con base en el conocimiento y la percepción propia a través de la adición de una red personal (Siemens, 2005).

Quizizz es una plataforma digital educativa que invita al estudiante a ser partícipe de divertidas actividades (Mac Namara \& Murphy, 2017). Además, se puede incluir memes que alertarán a los multijugadores, indicando si acertaron o no en cada una de las actividades asignadas por el docente, esto impulsa al estudiante a seguir compitiendo y atender las sesiones de clase (Zhao, 2019). A diferencia de otras aplicaciones, Quizizz posee una gama de avatares, fondo musical, memes y diversas recompensas para cada actividad realizada con éxito, lo cual influye fructíferamente en el aprendizaje del estudiante.

Como lo hace notar Gómez (2020) los estudiantes pueden adoptar una actitud proactiva y participativa durante una evaluación por medio de la herramienta Quizizz, así sea de uno de los cursos más aburridos para ellos. Esto se debe a los escenarios tecnológicos y dinámicos que proporciona la herramienta para favorecer el rendimiento académico del estudiante. Las afirmaciones anteriores señalan que además del uso satisfactorio que emana la plataforma Quizizz incentiva la motivación de los estudiantes.

Dicho en palabras de (Cueva Delgado et al., 2019) sostienen que la influencia del conectivismo, sobre el uso de las herramientas tecnológicas genera relaciones de impacto que posibilita la implementación de un proceso de enseñanza más activo, donde los principales protagonistas, los estudiantes, elevan sus niveles de motivación e interés por la investigación; a su vez, permite optimizar sus habilidades autónomas y creativas.

En la actualidad, según Valero Palomino \& Bullón Solís, (2021) las Tic son herramientas de mayor impacto en los diversos ámbitos, ya que intervienen de manera activa en el proceso enseñanza-aprendizaje, implementando tanto los puntos cuantitativos como cualitativos. Según (Coll et al., 2008) esto se debe a que hoy en día se tiene contacto directo con la tecnología, por consiguiente, los docentes se ven implicados en el trabajo de actualizarse constantemente para generar nuevas metodologías, con base en los diversos estilos de aprendizaje, puesto que las metodologías tradicionales obsoletas frente a las nuevas generaciones. Es por ello que se recurre a una nueva metodología, el conectivismo; que en concordancia con (Ovalles Pabón, 2014), es una manera efectiva de enseñar en esta era digital; dado que la tecnología ha captado mayor interés en la educación.

Inmersos en la situación que se vive, debido al contexto de virtualidad, la mayoría de los docentes de la Universidad Jorge Basadre Grohmann requieren aprender a utilizar diversas aplicaciones web en este contexto virtual de desafíos y retos en la enseñanza a nivel superior, donde los estudiantes demuestran excesiva falta de concentración, atención y bajo rendimiento académico, pero principalmente la escasa participación activa durante las sesiones virtuales, una de las principales causantes de estas problemáticas es la 
metodología tradicional aplicada en una sesión digital, una combinación que puede generar hastío en estudiantes en su formación académica profesional.

Con base en lo expuesto, las diversas plataformas educativas han ganado mayor realce y popularidad debido a los innumerables beneficios que aporta cada uno de ellos. En diferentes investigaciones Quispe et al., (2019); Soto \& Larenas, (2021); Laura et al., (2021); Purba, (2020) la herramienta Quizizz resalta entre algunas de ellas puesto que provoca una conexión significativa entre el educando y el educador. Quizizz puede ser aprovechada al máximo en las instituciones superiores, debido a que las clases son virtuales, por consiguiente, requieren de acceso al internet. Esta aplicación propicia la satisfacción de los agentes educativos; puesto que el docente tiene acceso a las respuestas de los estudiantes de manera inmediata y los estudiantes, además de ver sus errores, se retroalimentan constantemente, de ahí surge la popularidad de dicha plataforma.

En consecuencia, de lo mencionado, nace el presente estudio con el propósito de describir significativamente la percepción de Quizizz como parte del conectivismo en el aprendizaje a distancia en la educación superior del Perú en el año académico 2021 en su proceso, actitudinal, procedimental y conceptual, puesto que el estudiante se ve envuelto en esos tres momentos en el desarrollo del aprendizaje.

\section{TIPO Y DISEÑO DE LA INVESTIGACIÓN}

El presente estudio es cuantitativo bajo una investigación básica puesto que no busca solucionar un problema, por otra parte, servirá de base para la toma de mejores decisiones en un futuro acerca del aprendizaje del idioma inglés (Hernandez et al., 2016).

El diseño es no experimental, de nivel descriptivo ya que pretende identificar y diagnosticar la percepción de las personas, grupos o cualquier otro fenómeno sostenido a análisis (Hernandez et al., 2010).

\section{POBLACIÓN Y MUESTRA}

La muestra estuvo constituida por 160 estudiantes matriculados en el año académico 2021 de la carrera de Idioma Extranjero de la Universidad Nacional Jorge Basadre Grohmann de Tacna en el año académico 2021, considerando a todos los estudiantes de primero a quinto año de estudios a través de un muestro no aleatorio.

\section{INSTRUMENTO DE LA INVESTIGACIÓN}

El instrumento aplicado es un formulario basado en una escala de Likert, el cual en relación a la percepción de Quizizz como parte del conectivismo en el aprendizaje a distancia en la educación superior del Perú en el año académico 2021, presenta un grupo de enunciados en forma de afirmaciones a la percepción del estudiante, por otro lado, se centra en recopilar y diagnosticar la información rescatando las diversas percepciones que tenga el estudiante en su contexto natural.

En adición a lo mencionado, posibilita al estudiante escoger entre las siguientes opciones de la escala de Likert:

- Totalmente de acuerdo

- De acuerdo

- Neutral 
- $\quad$ En desacuerdo

- Totalmente en desacuerdo

Basado en los cuestionarios (Basuki y Hidayati, 2019). La confiabilidad de consistencia interna, fue a través de coeficiente de Alfa de Cronbach, obteniendo como resultado 0,92 lo que indica precisión en la medición.

Este mismo contiene las siguientes dimensiones:

\section{Tabla 1}

Dimensiones del instrumento

\begin{tabular}{lll}
\hline DIMENSIÓN & INDICADORES & ENUNCIADOS \\
\hline CONCEPTUAL & Aprendizaje Activo & $1,2,3,4,5$ \\
PROCEDIMENTAL & Herramientas Tecnológicas & $6,7,8,9$ \\
ACTITUDINAL & Apreciaciones del educando & $10,11,12,13$ \\
\hline
\end{tabular}

\section{RESULTADOS}

Dimensión 1: Conceptual

Indicador: Aprendizaje Activo

\section{Tabla 2}

Descripción de frecuencia sobre si ¿Considera indispensable el uso de las herramientas tecnológicas en su aprendizaje universitario? en estudiantes de la especialidad de Idioma extranjero de la Universidad Nacional Jorge Basadre Grohmann de la ciudad de Tacna-2021

\begin{tabular}{|c|c|c|c|c|c|c|c|c|c|c|c|c|}
\hline \multirow{3}{*}{$\begin{array}{l}\text { Considera usted } \\
\text { indispensable el uso de } \\
\text { las herramientas } \\
\text { tecnológicas en su } \\
\text { aprendizaje } \\
\text { universitario. }\end{array}$} & \multicolumn{12}{|c|}{ Año de estudio } \\
\hline & \multicolumn{2}{|c|}{ Primer } & \multicolumn{2}{|c|}{ Segundo } & \multicolumn{2}{|c|}{ Tercero } & \multicolumn{2}{|c|}{ Cuarto } & \multicolumn{2}{|c|}{ Quinto } & \multicolumn{2}{|r|}{ Cotal } \\
\hline & $\mathrm{n}$ & $\%$ & $\mathrm{n}$ & $\%$ & $\mathrm{n}$ & $\%$ & $\mathrm{n}$ & $\%$ & $\mathrm{n}$ & $\%$ & $\mathrm{n}$ & $\%$ \\
\hline Totalmente de acuerdo & 34 & 60.71 & 13 & 59.09 & 6 & 21.43 & 19 & 70.37 & 21 & 60.00 & 93 & 55.36 \\
\hline De acuerdo & 16 & 28.57 & 6 & 27.27 & 16 & 57.14 & 6 & 22.22 & 14 & 40.00 & 58 & 34.52 \\
\hline Neutral & 5 & 8.93 & 1 & 4.55 & 6 & 21.43 & 2 & 7.41 & 0 & 0.00 & 14 & 8.33 \\
\hline En desacuerdo & 1 & 1.79 & 1 & 4.55 & 0 & 0.00 & 0 & 0.00 & 0 & 0.00 & 2 & 1.19 \\
\hline Totalmente en desacuerdo & 0 & 0.00 & 1 & 4.55 & 0 & 0.00 & 0 & 0.00 & 0 & 0.00 & 1 & 0.60 \\
\hline Total & 56 & 100.0 & 22 & 100.00 & 28 & 100.00 & 27 & 100.00 & 35 & 100.00 & 168 & 100.00 \\
\hline
\end{tabular}

$\mathrm{X} 2=20.830 \mathrm{GL}=16$ P valor $=0.185$ No existe diferencia.

Fuente: Cuestionario dirigido a estudiantes de la muestra.

La tabla 2, es acerca de la relevancia que tiene las herramientas tecnológicas en el proceso de enseñanza de los estudiantes universitarios, se contempla que 168 estudiantes, que simboliza al $55.4 \%$ está totalmente de acuerdo que las herramientas tecnológicas son indispensables en el aprendizaje, mientras que el $0.6 \%$ está totalmente en desacuerdo en que sea fundamental el uso de estas herramientas. 
Debido al mundo globalizado en el que se vive, ya que la tecnología logra que en algunos aspectos hacer la vida más sencilla, es por ello que los estudiantes encuentran necesario el uso de herramientas tecnológicas para potencializar su aprendizaje académico universitario.

\section{Tabla 3}

Descripción de frecuencia sobre si ¿La aplicación Quirizz genera participación activa en el aprendizaje? En estudiantes de la especialidad de Idioma extranjero de la Universidad Nacional Jorge Basadre Grohmann de la ciudad de Tacna del año 2021.

\begin{tabular}{|c|c|c|c|c|c|c|c|c|c|c|c|c|}
\hline \multirow{3}{*}{$\begin{array}{l}\text { La aplicación } \\
\text { Quizizz } \\
\text { genera } \\
\text { participación } \\
\text { activa en el } \\
\text { aprendizaje. }\end{array}$} & \multicolumn{12}{|c|}{ Año de estudio } \\
\hline & \multicolumn{2}{|c|}{ Primer } & \multicolumn{2}{|c|}{ Segundo } & \multicolumn{2}{|c|}{ Tercero } & \multicolumn{2}{|c|}{ Cuarto } & \multicolumn{2}{|c|}{ Quinto } & \multicolumn{2}{|r|}{ Total } \\
\hline & $\mathrm{n}$ & $\%$ & $\mathrm{n}$ & $\%$ & $\mathrm{n}$ & $\%$ & $\mathrm{n}$ & $\%$ & $\mathrm{n}$ & $\%$ & $\mathrm{n}$ & $\%$ \\
\hline $\begin{array}{l}\text { Totalmente de } \\
\text { acuerdo }\end{array}$ & 10 & 17.86 & 9 & 40.91 & 6 & 21.43 & 7 & 25.93 & 16 & 45.71 & 48 & 28.57 \\
\hline De acuerdo & 37 & 66.07 & 10 & 45.45 & 16 & 57.14 & 14 & 51.85 & 16 & 45.71 & 93 & 55.36 \\
\hline Neutral & 8 & 14.29 & 3 & 13.64 & 6 & 21.43 & 6 & 22.22 & 2 & 5.71 & 25 & 14.88 \\
\hline En desacuerdo & 0 & 0.00 & 0 & 0.00 & 0 & 0.00 & 0 & 0.00 & 1 & 2.86 & 1 & 0.60 \\
\hline $\begin{array}{l}\text { Totalmente en } \\
\text { desacuerdo }\end{array}$ & 1 & 1.79 & 0 & 0.00 & 0 & 0.00 & 0 & 0.00 & 0 & 0.00 & 1 & 0.60 \\
\hline Total & 56 & 100.00 & 22 & 100.00 & 28 & 100.00 & 27 & 100.00 & 35 & 100.00 & 168 & 100.00 \\
\hline
\end{tabular}

La tabla 3 muestra la relevancia que tiene las herramientas tecnológicas en el proceso de aprendizaje de los estudiantes universitarios, se contempla que 168 estudiantes, que simboliza al $55.34 \%$ está totalmente de acuerdo que las herramientas tecnológicas son indispensables en el aprendizaje, mientras que el $0.6 \%$ está totalmente en desacuerdo en que sea fundamental el uso de estas herramientas.

La mayor parte de estudiantes se muestran de acuerdo frente a la participación activa que propaga la herramienta Quizizz, debido a lo que respecta la herramienta digital presenta una interfaz muy atractiva y emocionante. Sin embargo, un porcentaje reducido no está de acuerdo con lo dicho con anterioridad.

\section{Tabla 4}

Descripción de frecuencia sobre si ¿La aplicación Quiziž genera participación activa en el aprendizaje? En estudiantes de la especialidad de Idioma extranjero de la Universidad Nacional Jorge Basadre Grohmann de la ciudad de Tacna-2021

\begin{tabular}{|c|c|c|c|c|c|c|c|c|c|c|c|c|}
\hline \multirow{3}{*}{$\begin{array}{l}\text { La aplicación Quizizz } \\
\text { genera participación } \\
\text { activa en el } \\
\text { aprendizaje. }\end{array}$} & \multicolumn{12}{|c|}{ Año de estudio } \\
\hline & \multicolumn{2}{|c|}{ Primer } & \multicolumn{2}{|c|}{ Segundo } & \multicolumn{2}{|c|}{ Tercero } & \multicolumn{2}{|c|}{ Cuarto } & \multicolumn{2}{|c|}{ Quinto } & \multicolumn{2}{|c|}{ Total } \\
\hline & $\mathrm{n}$ & $\%$ & $\mathrm{n}$ & $\%$ & $\mathrm{n}$ & $\%$ & $\mathrm{n}$ & $\%$ & $\mathrm{n}$ & $\%$ & $\mathrm{n}$ & $\%$ \\
\hline $\begin{array}{l}\text { Totalmente de } \\
\text { acuerdo }\end{array}$ & 0 & 7.86 & & 0.91 & & 1.43 & & 5.93 & 6 & 5.71 & 8 & 8.57 \\
\hline
\end{tabular}




\begin{tabular}{|c|c|c|c|c|c|c|c|c|c|c|c|c|}
\hline De acuerdo & 7 & 6.07 & 0 & .5 .45 & 6 & 7.14 & 4 & 1.85 & 6 & 5.71 & 3 & 5.36 \\
\hline Neutral & & 4.29 & & 3.64 & & 1.43 & & 2.22 & & .71 & .5 & 4.88 \\
\hline En desacuerdo & 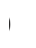 & .00 & & 1.00 & 1 & .00 & 1 & 1.00 & & .86 & & .60 \\
\hline $\begin{array}{l}\text { Totalmente } \\
\text { en desacuerdo }\end{array}$ & & .79 & & 1.00 & 1 & .00 & 1 & 1.00 & 1 & .00 & & .60 \\
\hline Total & 6 & 00.00 & 2 & 00.00 & 8 & 00.00 & 7 & 00.00 & 5 & 00.00 & 68 & 00.00 \\
\hline
\end{tabular}

$\mathrm{X} 2=19.400 \mathrm{GL}=16 \mathrm{P}$ valor $=0.248$ No existe diferencia.

Fuente: Cuestionario dirigido a estudiantes de la muestra.

En la tabla 04, aludida a la participación activa genera la participación a través de Quizizz en los estudiantes, se puede registrar el 55.4\% que compete a 48 estudiantes, se encuentran de acuerdo en que dicha herramienta genera intervención de los estudiantes, por otro lado, un solo estudiante que representa el $0.6 \%$ del total, considera que Quizizz no ocasiona participación activa.

La mayor parte de estudiantes se muestran de acuerdo frente a la participación activa que propaga la herramienta Quizizz, debido a que la herramienta digital presenta una interfaz muy atractiva y emocionante. Sin embargo, un porcentaje reducido no está de acuerdo con lo dicho con anterioridad.

\section{Tabla 5}

Descripción de frecuencia sobre si ¿Las actividades de Quirizz contribuyen a comprobar los conocimientos impartidos en clases? En estudiantes de la especialidad de Idioma extranjero de la Universidad Nacional Jorge Basadre Grohmann de la ciudad de Tacna del año 2021.

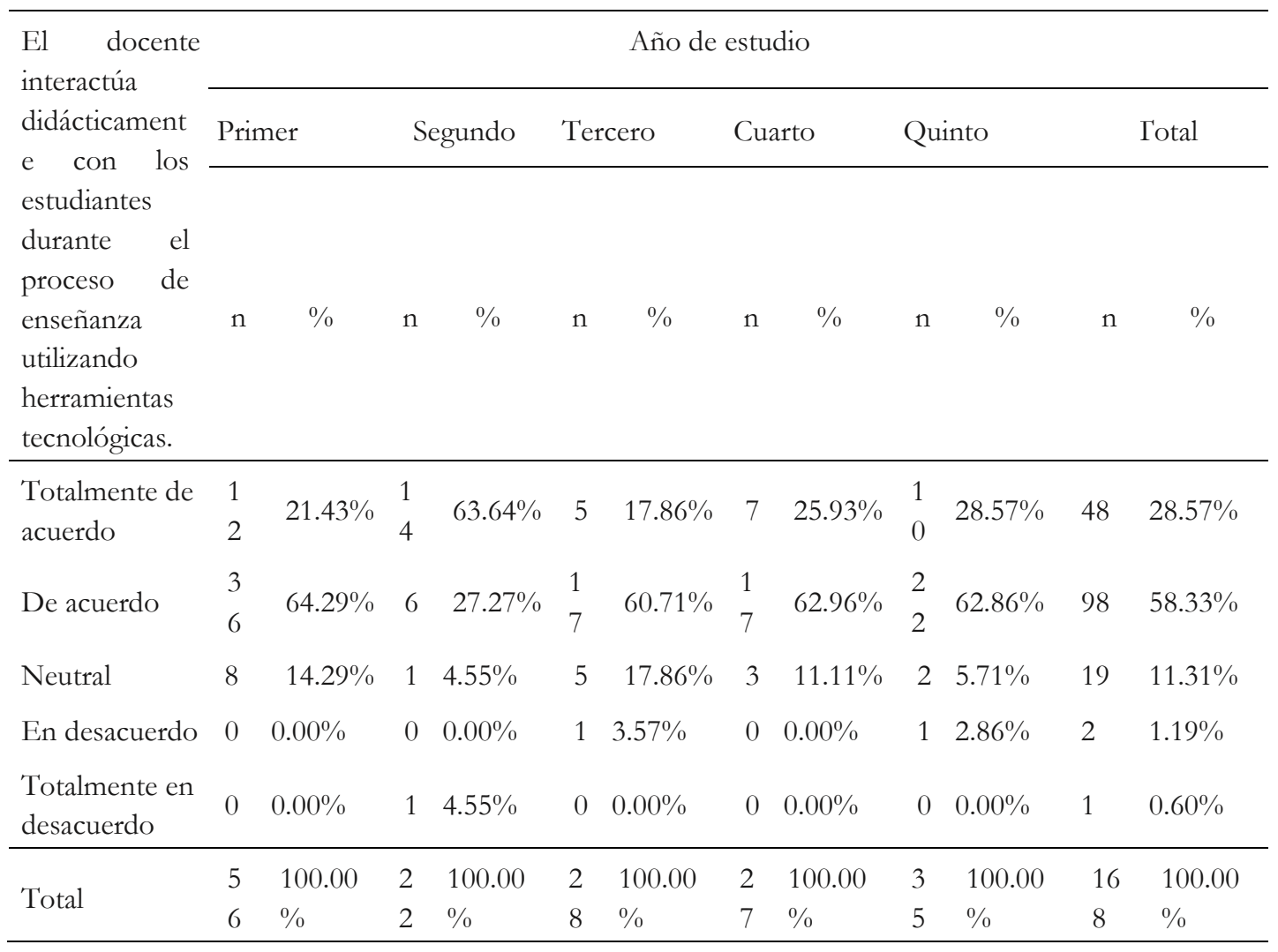


$\mathrm{X} 2=29.283 \mathrm{GL}=16 \mathrm{P}$ valor $=0.022 \mathrm{Si}$ existe diferencia

Fuente: Cuestionario dirigido a estudiantes de la muestra.

La tabla 5 se encuentra relacionada con la opinión que tienen los estudiantes acerca del papel que cumple el docente con las herramientas tecnológicas. El 58.3\% de estudiantes dan a entender que el docente sabe manejar eficazmente herramientas tecnológicas, el $0.6 \%$ piensa que el profesor no puede comunicarse correctamente durante el aprendizaje utilizando dichas herramientas.

Según la percepción de los estudiantes, la mayoría de docentes utilizan progresivamente las herramientas tecnológicas satisfactoriamente para facilitar el proceso de enseñanza. Lo cual indica, que docentes necesitan aun seguir actualizando sus competencias tecnológicas para complementar y lograr los objetivos propuestos de cada curso.

\section{Tabla 6}

Descripción de frecuencia sobre si ¿Las actividades de Quizizz contribuyen a comprobar los conocimientos impartidos en clases? En estudiantes de la especialidad de Idioma extranjero de la Universidad Nacional Jorge Basadre Grohmann de la ciudad de Tacna-2021

\begin{tabular}{|c|c|c|c|c|c|c|c|c|c|c|c|c|}
\hline \multirow{3}{*}{$\begin{array}{l}\text { Las actividades de } \\
\text { Quizizz contribuyen } \\
\text { a comprobar los } \\
\text { conocimientos } \\
\text { impartidos en clases. }\end{array}$} & \multicolumn{12}{|c|}{ Año de estudio } \\
\hline & \multicolumn{2}{|c|}{ Primer } & \multicolumn{2}{|c|}{ Segundo } & \multicolumn{2}{|c|}{ Tercero } & \multicolumn{2}{|c|}{ Cuarto } & \multicolumn{2}{|c|}{ Quinto } & \multicolumn{2}{|c|}{ Total } \\
\hline & $\mathrm{n}$ & $\%$ & $\mathrm{n}$ & $\%$ & $\mathrm{n}$ & $\%$ & $\mathrm{n}$ & $\%$ & $\mathrm{n}$ & $\%$ & $\mathrm{n}$ & $\%$ \\
\hline $\begin{array}{l}\text { Totalmente de } \\
\text { acuerdo }\end{array}$ & 11 & 19.64 & 11 & 50.00 & 6 & 21.43 & 8 & 29.63 & 15 & 42.86 & 51 & 30.36 \\
\hline De acuerdo & 36 & 64.29 & 7 & 31.82 & 14 & 50.00 & 12 & 44.44 & 17 & 48.57 & 86 & 51.19 \\
\hline Neutral & 9 & 16.07 & 3 & 13.64 & 8 & 28.57 & 6 & 22.22 & 3 & 8.57 & 29 & 17.26 \\
\hline En desacuerdo & 0 & 0.00 & 0 & 0.00 & 0 & 0.00 & 0 & 0.00 & 0 & 0.00 & 0 & 0.00 \\
\hline $\begin{array}{l}\text { Totalmente en } \\
\text { desacuerdo }\end{array}$ & 0 & 0.00 & 1 & 4.55 & 0 & 0.00 & 1 & 3.70 & 0 & 0.00 & 2 & 1.19 \\
\hline Total & 56 & 100.00 & 22 & 100.00 & 28 & 100.00 & 27 & 100.00 & 35 & 100.00 & 168 & 100.00 \\
\hline
\end{tabular}

$\mathrm{X} 2=20.373 \mathrm{GL}=16 \mathrm{P}$ valor $=0.060$ No existe diferencia.

Fuente: Cuestionario dirigido a estudiantes de la muestra.

La tabla 6 está referida acerca de si las actividades de la herramienta Quizizz contribuyen con la retroalimentación. El 51.1\% afirma estar de acuerdo que dicha herramienta es beneficioso al momento de la retroalimentación. Por el contrario, el 1.2\% es totalmente en desacuerdo, en que Quizizz pueda ser de utilidad para corroborar lo aprendido en clases. 
Los estudiantes muestran una actitud positiva frente al uso de Quizizz, considerando los elementos de gamificación que evidencia la comodidad del estudiante al aprender y el factor motivación que fuerza el proceso de aprendizaje de los estudiantes.

\section{Tabla 7}

Descripción de frecuencia sobre si ¿La utilización de las herramientas tecnológicas en clase favorece a la retroalimentación del aprendizaje? En estudiantes de la especialidad de Idioma extranjero de la Universidad Nacional Jorge Basadre Grohmann de la ciudad de Tacna-2021

\begin{tabular}{|c|c|c|c|c|c|c|c|c|c|c|c|c|}
\hline \multirow{3}{*}{$\begin{array}{l}\text { La utilización de } \\
\text { las herramientas } \\
\text { tecnológicas en } \\
\text { clase favorece a la } \\
\text { retroalimentación } \\
\text { del aprendizaje. }\end{array}$} & \multicolumn{12}{|c|}{ Año de estudio } \\
\hline & \multicolumn{2}{|c|}{ Primer } & \multicolumn{2}{|c|}{ Segundo } & \multicolumn{2}{|c|}{ Tercero } & \multicolumn{2}{|c|}{ Cuarto } & \multicolumn{2}{|c|}{ Quinto } & \multicolumn{2}{|l|}{ Total } \\
\hline & $\mathrm{n}$ & $\%$ & $\mathrm{n}$ & $\%$ & $\mathrm{n}$ & $\%$ & $\mathrm{n}$ & $\%$ & $\mathrm{n}$ & $\%$ & $\mathrm{n}$ & $\%$ \\
\hline Totalmente de acuerdo & 24 & 42.86 & 12 & 54.55 & 7 & 25.00 & 12 & 44.44 & 13 & 37.14 & 68 & 40.48 \\
\hline De acuerdo & 30 & 53.57 & 9 & 40.91 & 18 & 64.29 & 12 & 44.44 & 20 & 57.14 & 89 & 52.98 \\
\hline Neutral & 2 & 3.57 & 0 & 0.00 & 3 & 10.71 & 1 & 3.70 & 2 & 5.71 & 8 & 4.76 \\
\hline En desacuerdo & 0 & 0.00 & 0 & 0.00 & 0 & 0.00 & 1 & 3.70 & 0 & 0.00 & 1 & 0.60 \\
\hline $\begin{array}{l}\text { Totalmente en } \\
\text { desacuerdo }\end{array}$ & 0 & 0.00 & 1 & 4.55 & 0 & 0.00 & 1 & 3.70 & 0 & 0.00 & 2 & 1.19 \\
\hline Total & 56 & 100.00 & 22 & 100.00 & 28 & 100.00 & 27 & 100.00 & 35 & 100.00 & 168 & 100.00 \\
\hline
\end{tabular}

$\mathrm{X} 2=18.362 \mathrm{GL}=16$ P valor $=0.303$ No existe diferencia.

Fuente: Cuestionario dirigido a estudiantes de la muestra.

En la tabla 7, con relación al momento de la retroalimentación y las herramientas tecnológicas y que como juntas contribuyen en la enseñanza, se percibe que el $53 \%$ de los estudiantes encuestados indican estar total de acuerdo en que dichas herramientas, no obstante $0.6 \%$ señala que las herramientas tecnológicas no cooperan con la retroalimentación.

Los beneficios de la mencionada herramienta tecnología permite la retroalimentación asíncrona al revisar las respuestas al finalizar los ejercicios propuestos y sincrónicamente al socializar el aprendizaje en el aula. Lo cual permite reforzar el aprendizaje autónomo del estudiante y reflexión constante en su aprendizaje.

Dimensión 2: Procedimental

Indicador: Herramientas Tecnológicas

\section{Figura 1}

Descripción de frecuencia sobre si ¿El docente hace uso de herramientas tecnológicas para socializar el conocimiento y fortalecer el aprendizaje significativo en clase? En estudiantes de la especialidad de Idioma extranjero de la Universidad Nacional Jorge Basadre Grohmann de la ciudad de Tacna-2021 


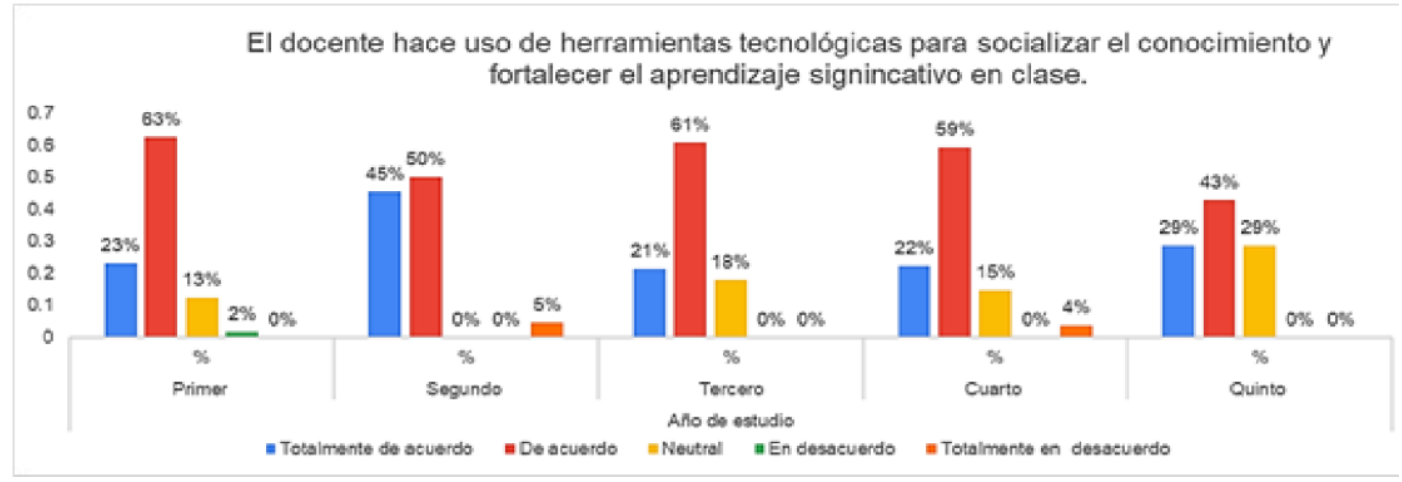

$\mathrm{X} 2=20.131 \mathrm{GL}=16 \mathrm{P}$ valor $=0.214$ No existe diferencia.

Fuente: Cuestionario dirigido a estudiantes de la muestra.

En la figura 1, se refiere al papel que el docente tiene al saber utilizar las herramientas digitales para trabajar los conocimientos que el alumno trae de experiencias anteriores y así construir nuevos, se aprecia que el 56\% de los estudiantes parecen estar con que el docente se apoya de dichas herramientas para fortificar el aprendizaje significativo. Por el contrario, el $0.6 \%$ de los encuestados que representa a un estudiante, indica que el docente no corrobora su enseñanza con las herramientas digitales.

Un gran número de encuestados evidencian que el docente da a conocer la información por las herramientas tecnológicas, en vista de que a los estudiantes de hoy en día la tecnología es parte de sus vidas, así como por medio de ellas la información les es más impactante. Aunque un reducido porcentaje refleja que el docente no ha hecho uso de estas herramientas o si las hace parece no cumplir con socializar el conocimiento.

\section{Figura 2}

Descripción de frecuencia sobre si ¿El docente guia y entrena en el uso de herramienta Quirizz para hacer uso de la misma de manera autónoma? En estudiantes de la especialidad de Idioma extranjero de la Universidad Nacional Jorge Basadre Grohmann de la ciudad de Tacna-2021

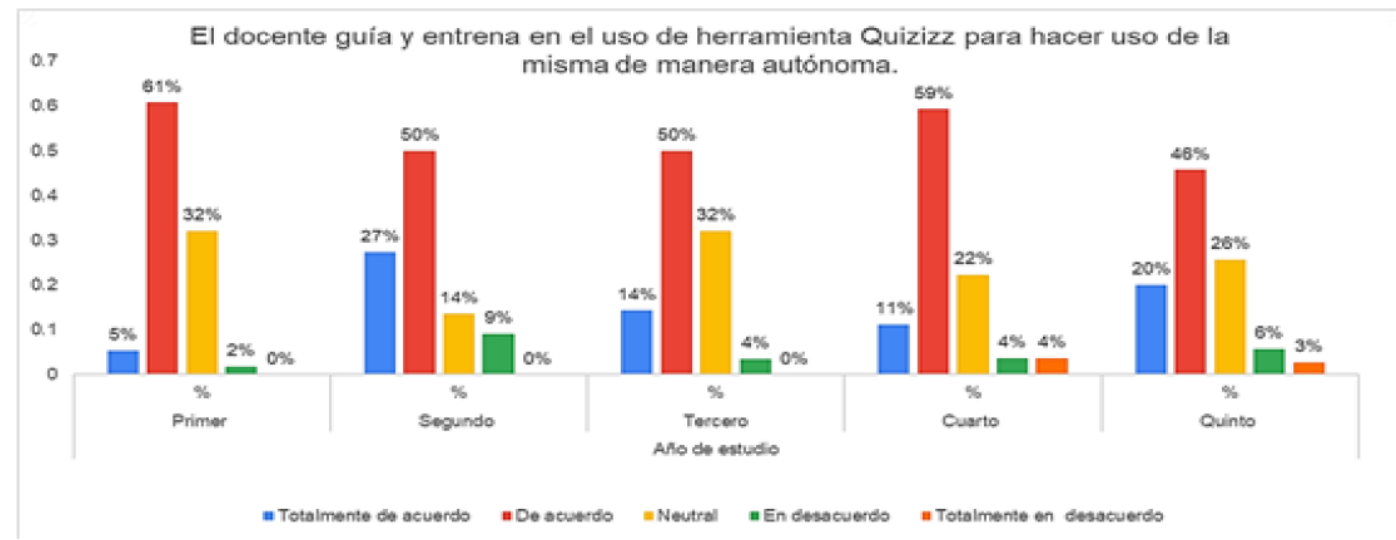

$\mathrm{X} 2=16.493 \mathrm{GL}=16 \mathrm{P}$ valor $=0.419$ No existe diferencia.

Fuente: Cuestionario dirigido a estudiantes de la muestra.

En la figura 2, con alusión a la guía hecha por el docente para el uso correcto de la herramienta Quizizz, en ella se puede apreciar que el 54.17\% de los estudiantes están de acuerdo con que el docente se desenvuelve de manera óptima en lo que se refiere al 
manejo de las herramientas digitales, para compartirlo con los estudiantes. Por otro lado, el $1.2 \%$ no piensa de la misma manera.

La gran mayoría de los encuestados consideran que el docente enseña lo aprendido del manejo de la herramienta Quizizz de manera óptima, esto se debe a que hoy en día los docentes se han visto en la necesidad de recurrir a las herramientas digitales, para lograr un aprendizaje didáctico y participativo, sin embrago, un porcentaje no está de acuerdo con que el docente pueda guiar a los estudiantes en el manejo de dicha herramienta, esto es debido a que el docente no está lo suficiente empalmado con esta.

\section{Figura 3}

Descripción de frecuencia sobre si ¿Durante el desarrollo de las diversas actividades de Quizizz ha tenido usted menos dificultades en su aprendizaje que cuando trabajabas sin ella? En estudiantes de la especialidad de Idioma extranjero de la Universidad Nacional Jorge Basadre Grohmann de la ciudad de Tacna-2021

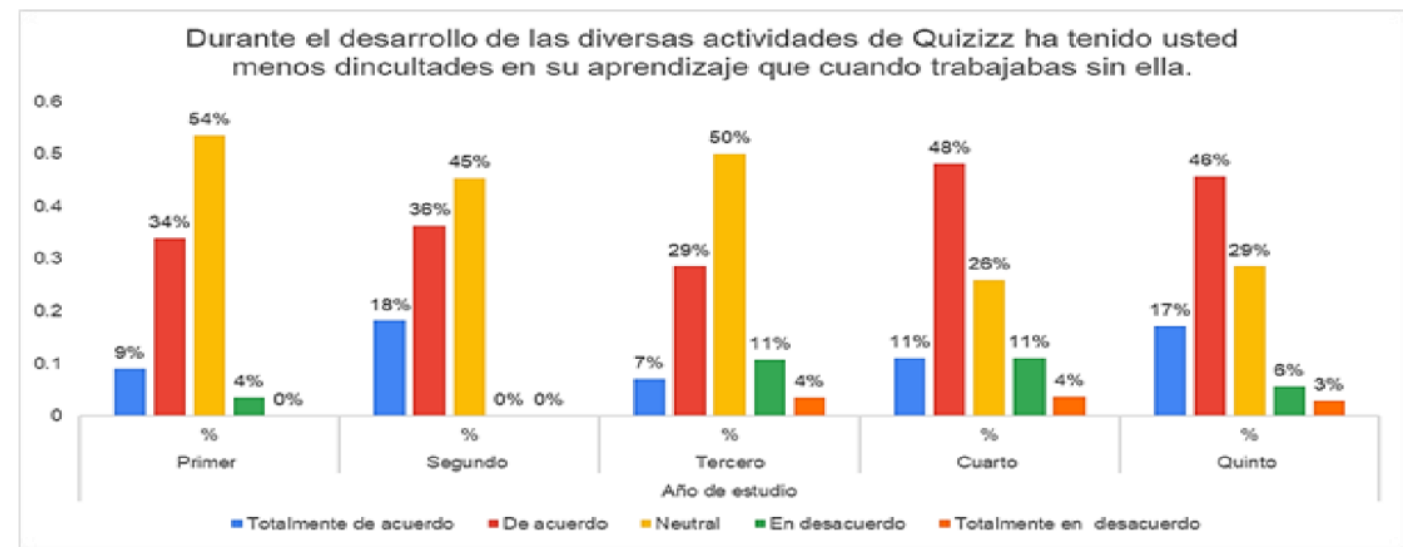

$\mathrm{X} 2=16.884 \mathrm{GL}=16 \mathrm{P}$ valor $=0.393$ No existe diferencia.

Fuente: Cuestionario dirigido a estudiantes de la muestra.

En la figura 3, se alude a los desempeños que los estudiantes han tenido luego del uso del uso de Quizizz, se puede observar que al $42.3 \%$ no parece haber impactado mucho ya que mantienen neutrales en cuanto el aprendizaje con Quizizz y sin ella. Si bien el $1.8 \%$ piensa que no influye mucho la mencionada plataforma en su aprendizaje, puesto que tienen las mismas dificultades en su proceso de aprendizaje cuando trabaja con Quizizz como sin ella.

El gran porcentaje parece ecuánime en cuanto si Quizizz ayuda con las dificultades encontradas en el aprendizaje. Con respecto a lo anterior, la enseñanza no es algo que solo deba recaer en manos de las herramientas digitales si bien es cierto que estas ayudan, pero el docente junto al estudiante debe de trabajar de la para que el aprendizaje no presente dificultades.

\section{Figura 4}

Descripción de frecuencia sobre si ¿Los cuestionarios de Quizizz contribuyen a lograr un mayor manejo de conocimiento y contenidos propuestos del curso? En estudiantes de la especialidad de Idioma extranjero de la Universidad Nacional Jorge Basadre Grohmann de la ciudad de Tacna-2021 


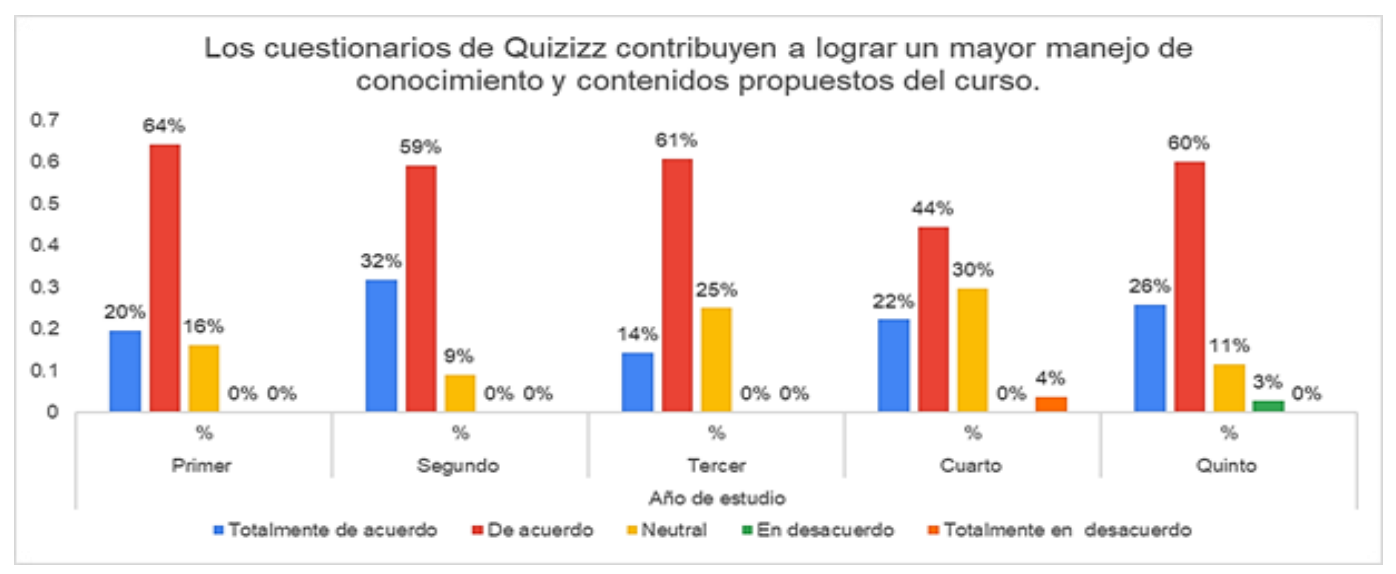

$\mathrm{X} 2=17.111 \mathrm{GL}=16 \mathrm{P}$ valor $=0.378$ No existe diferencia.

Fuente: Cuestionario dirigido a estudiantes de la muestra.

La figura 4 hace referencia a la contribución de la herramienta Quizizz puede proporcionar a lo que se refiere utilizar los conocimientos y contenidos dados en el curso, se contempla que el $59 \%$ de los estudiantes se encuentran de acuerdo que los cuestionarios, que ofrece dicha herramienta en cuestión, colaboran con los contenidos planteados en el curso, en cambio el $0.6 \%$ piensa lo contrario a lo mencionado anteriormente.

La mayoría de los estudiantes encuestados siente que los cuestionarios que brinda Quizizz les ayuda en cuanto al manejo de información del contenido, puesto que el docente puede formular de manera resumida y específica en forma de pregunta o afirmación cada tema, subtema, concepto, etc. Sin embargo, hay un grupo pequeño de estudiantes sienten que dichos cuestionarios no les contribuyen a entender el contenido del curso.

Dimensión 3: Actitudinal

Indicador: Apreciaciones del educando

\section{Tabla 8}

Descripción de frecuencia sobre si ¿Quizizz es emocionante, interesante, motivador y divertido? En estudiantes de la especialidad de Idioma extranjero de la Universidad Nacional Jorge Basadre Grohmann de la ciudad de Tacna-2021

\begin{tabular}{|c|c|c|c|c|c|c|c|c|c|c|c|c|}
\hline \multirow{3}{*}{$\begin{array}{l}\text { Quizizz es } \\
\text { emocionante, } \\
\text { interesante, } \\
\text { motivador y } \\
\text { divertido. }\end{array}$} & \multicolumn{12}{|c|}{ Año de estudio } \\
\hline & \multicolumn{2}{|c|}{ Primer } & \multicolumn{2}{|c|}{ Segundo } & \multicolumn{2}{|c|}{ Tercero } & \multicolumn{2}{|c|}{ Cuarto } & \multicolumn{2}{|c|}{ Quinto } & \multicolumn{2}{|c|}{ Total } \\
\hline & $\mathrm{n}$ & $\%$ & $\mathrm{n}$ & $\%$ & $\mathrm{n}$ & $\%$ & $\mathrm{n}$ & $\%$ & $\mathrm{n}$ & $\%$ & $\mathrm{n}$ & $\%$ \\
\hline $\begin{array}{l}\text { Totalmente de } \\
\text { acuerdo }\end{array}$ & 12 & 21.43 & 7 & 31.82 & 8 & 28.57 & 15 & 55.56 & 17 & 48.57 & 59 & 35.12 \\
\hline De acuerdo & 27 & 48.21 & 14 & 63.64 & 11 & 39.29 & 7 & 25.93 & 12 & 34.29 & 71 & 42.26 \\
\hline Neutral & 16 & 28.57 & 1 & 4.55 & 8 & 28.57 & 5 & 18.52 & 4 & 11.43 & 34 & 20.24 \\
\hline En desacuerdo & 1 & 1.79 & 0 & 0.00 & 1 & 3.57 & 0 & 0.00 & 1 & 2.86 & 3 & 1.79 \\
\hline
\end{tabular}




\begin{tabular}{lccccccccccccc}
\hline $\begin{array}{l}\text { Totalmente en } \\
\text { desacuerdo }\end{array}$ & 0 & 0.00 & 0 & 0.00 & 0 & 0.00 & 0 & 0.00 & 1 & 2.86 & 1 & 0.60 \\
\begin{tabular}{l} 
Total \\
\hline
\end{tabular} & 56 & 100.00 & 22 & 100.00 & 28 & 100.00 & 27 & 100.00 & 35 & 100.00 & 168 & 100.00 \\
\hline
\end{tabular}

$\mathrm{X} 2=25.892 \mathrm{GL}=16 \mathrm{P}$ valor $=0.056$ No existe diferencia.

Fuente: Cuestionario dirigido a estudiantes de la muestra.

La tabla 8 refiere a las estimaciones que tiene el estudiante hacia la herramienta digital, Quizizz. Se observa que 71 personas que representan el $42.2 \%$ califican de una manera positiva a dicha herramienta; por otro lado, el $0.6 \%$ considera que Quizizz no es divertido ni emocionante.

Lo indicado por la mayoría de los estudiantes se debe a que la configuración de herramienta en cuestión cuenta con las opciones de música, asimismo, memes que harán que los estudiantes se diviertan con lo que hacen, y se sientan motivados a seguir con el aprendizaje.

\section{Tabla 9}

Descripción de frecuencia sobre si ¿La aplicación Quizizz le ba ayudado a reconocer sus fortalezas y debilidades al realizar diversas actividades? En estudiantes de la especialidad de Idioma extranjero de la Universidad Nacional Jorge Basadre Grohmann de la ciudad de Tacna-2021

\begin{tabular}{|c|c|c|c|c|c|c|c|c|c|c|c|c|}
\hline \multirow{3}{*}{$\begin{array}{lr}\text { La aplicación } \\
\text { Quizizz le ha } \\
\text { ayudado } & \text { a } \\
\text { reconocer } & \text { sus } \\
\text { fortalezas } & \text { y } \\
\text { debilidades } & \text { al } \\
\text { realizar diversas } \\
\text { actividades. }\end{array}$} & \multicolumn{12}{|c|}{ Año de estudio } \\
\hline & \multicolumn{2}{|c|}{ Primer } & \multicolumn{2}{|c|}{ Segundo } & \multicolumn{2}{|c|}{ Tercero } & \multicolumn{2}{|c|}{ Cuarto } & \multicolumn{2}{|c|}{ Quinto } & \multicolumn{2}{|c|}{ Total } \\
\hline & $\mathrm{n}$ & $\%$ & $\mathrm{n}$ & $\%$ & $\mathrm{n}$ & $\%$ & $\mathrm{n}$ & $\%$ & $\mathrm{n}$ & $\%$ & $\mathrm{n}$ & $\%$ \\
\hline $\begin{array}{l}\text { Totalmente de } \\
\text { acuerdo }\end{array}$ & 7 & 12.50 & 5 & 22.73 & 5 & 17.86 & 9 & 33.33 & 12 & 34.29 & 38 & 22.62 \\
\hline De acuerdo & 29 & 51.79 & 15 & 68.18 & 14 & 50.00 & 11 & 40.74 & 19 & 54.29 & 88 & 52.38 \\
\hline Neutral & 20 & 35.71 & 2 & 9.09 & 7 & 25.00 & 5 & 18.52 & 1 & 2.86 & 35 & 20.83 \\
\hline En desacuerdo & 0 & 0.00 & 0 & 0.00 & 2 & 7.14 & 2 & 7.41 & 3 & 8.57 & 7 & 4.17 \\
\hline $\begin{array}{l}\text { Totalmente en } \\
\text { desacuerdo }\end{array}$ & 0 & 0.00 & 0 & 0.00 & 0 & 0.00 & 0 & 0.00 & 0 & 0.00 & 0 & 0.00 \\
\hline Total & 56 & 100.00 & 22 & 100.00 & 28 & 100.00 & 27 & 100.00 & 35 & 100.00 & 168 & 100.00 \\
\hline
\end{tabular}

$\mathrm{X} 2=27.393 \mathrm{GL}=16 \mathrm{P}$ valor $=0.007 \mathrm{Si}$ existe diferencia.

Fuente: Cuestionario dirigido a estudiantes de la muestra.

En la tabla 9 se refiere al impacto que tiene la herramienta Quizizz en los estudiantes, el $52.3 \%$ que representa a 88 estudiantes está de acuerdo con que la aplicación le ha sido de utilidad a la hora del diagnóstico del desempeño en actividades realizadas en clase. 
La mayoría de los estudiantes tienen en cuenta que a través de la herramienta Quizizz pueden conocer las debilidades y fortalezas que tienen a la hora de realizar diversas actividades, gran medida se debe a que Quizizz permite mostrar si lo que se ha respondido está bien o no al instante, así como su resumen una vez terminado el cuestionario, esto es de ayuda para mejorar y repasar las falencias en las actividades.

\section{Tabla 10}

Descripción de frecuencia sobre si ¿Considera usted que la aplicación Quizizz. incentiva a seguir aprendiendo por sus componentes de retroalimentación y de juego? En estudiantes de la especialidad de Idioma extranjero de la Universidad Nacional Jorge Basadre Grohmann de la ciudad de Tacna-2021

\begin{tabular}{|c|c|c|c|c|c|c|c|c|c|c|c|c|}
\hline \multirow{3}{*}{$\begin{array}{l}\text { ¿Considera usted que } \\
\text { la aplicación Quizizz } \\
\text { incentiva a seguir } \\
\text { aprendiendo por sus } \\
\text { componentes de } \\
\text { retroalimentación y } \\
\text { de juego? }\end{array}$} & \multicolumn{12}{|c|}{ Año de estudio } \\
\hline & \multicolumn{2}{|c|}{ Primer } & \multicolumn{2}{|c|}{ Segundo } & \multicolumn{2}{|c|}{ Tercero } & \multicolumn{2}{|c|}{ Cuarto } & \multicolumn{2}{|c|}{ Quinto } & \multicolumn{2}{|c|}{ Total } \\
\hline & $\mathrm{n}$ & $\%$ & $\mathrm{n}$ & $\%$ & $\mathrm{n}$ & $\%$ & $\mathrm{n}$ & $\%$ & $\mathrm{n}$ & $\%$ & $\mathrm{n}$ & $\%$ \\
\hline $\begin{array}{l}\text { Totalmente de } \\
\text { acuerdo }\end{array}$ & 11 & 19.64 & 6 & 27.27 & 4 & 14.29 & 10 & 37.04 & 10 & 28.57 & 41 & 24.40 \\
\hline De acuerdo & 35 & 62.50 & 14 & 63.64 & 20 & 71.43 & 11 & 40.74 & 21 & 60.00 & 101 & 60.12 \\
\hline Neutral & 9 & 16.07 & 2 & 9.09 & 4 & 14.29 & 6 & 22.22 & 3 & 8.57 & 24 & 14.29 \\
\hline En desacuerdo & 1 & 1.79 & 0 & 0.00 & 0 & 0.00 & 0 & 0.00 & 1 & 2.86 & 2 & 1.19 \\
\hline $\begin{array}{l}\text { Totalmente en } \\
\text { desacuerdo }\end{array}$ & 0 & 0.00 & 0 & 0.00 & 0 & 0.00 & 0 & 0.00 & 0 & 0.00 & 0 & 0.00 \\
\hline Total & 56 & 100.00 & 22 & 100.00 & 28 & 100.00 & 27 & 100.00 & 35 & 100.00 & 168 & $\begin{array}{l}100.0 \\
0\end{array}$ \\
\hline
\end{tabular}

$\mathrm{X} 2=10.595 \mathrm{GL}=16 \mathrm{P}$ valor $=0.564$ No existe diferencia.

Fuente: Cuestionario dirigido a estudiantes de la muestra.

La tabla 10 está relacionada con la consideración que tienen los estudiantes frente al rol incitativo que desenvuelve la herramienta Quizizz para seguir aprendiendo gracias a sus componentes de retroalimentación y de juego que esta misma proporciona. Se aprecia que el $60.12 \%$ conformado por 101 estudiantes muestran una posición acorde a la función estimulante que genera Quizizz para que sigan aprendiendo; por otro lado, existe una minoría del $1.19 \%$ que no estima el rol motivador de la herramienta en mención.

Con base a lo mencionado, se demuestra que la mayor parte de los estudiantes encuestados aceptan que Quizizz es una herramienta muy inspiradora que impulsa a continuar aprendiendo de manera activa y constante, lo cual es gratificante para su proceso de aprendizaje. Sin embargo, se pudo establecer la existencia de un limitado número de estudiantes que no aprecian la función estimulante de Quizizz; lo cual es preocupante.

\section{Tabla 11}

Descripción de frecuencia sobre si ¿Considera usted que ha mejorado su concentración al momento de desarrollar los cuestionarios propuestos de Quizizz? En estudiantes de la especialidad de Idioma extranjero de la Universidad Nacional Jorge Basadre Grohmann de la ciudad de Tacna-2021 


\begin{tabular}{|c|c|c|c|c|c|c|c|c|c|c|c|c|}
\hline \multirow{3}{*}{$\begin{array}{l}\text { ¿Considera usted } \\
\text { que ha mejorado su } \\
\text { concentración al } \\
\text { momento de desa- } \\
\text { rrollar los } \\
\text { cuestionarios } \\
\text { propuestos de } \\
\text { Quizizz? }\end{array}$} & \multicolumn{12}{|c|}{ Año de estudio } \\
\hline & \multicolumn{2}{|c|}{ Primer } & \multicolumn{2}{|c|}{ Segundo } & \multicolumn{2}{|c|}{ Tercero } & \multicolumn{2}{|c|}{ Cuarto } & \multicolumn{2}{|c|}{ Quinto } & \multicolumn{2}{|c|}{ Total } \\
\hline & $\mathrm{n}$ & $\%$ & $\mathrm{n}$ & $\%$ & $\mathrm{n}$ & $\%$ & $\mathrm{n}$ & $\%$ & $\mathrm{n}$ & $\%$ & $\mathrm{n}$ & $\%$ \\
\hline $\begin{array}{l}\text { Totalmente de } \\
\text { acuerdo }\end{array}$ & 13 & 23.21 & 6 & 27.27 & 3 & 10.71 & 10 & 37.04 & 9 & 25.71 & 41 & 24.40 \\
\hline De acuerdo & 22 & 39.29 & 12 & 54.55 & 11 & 39.29 & 9 & 33.33 & 17 & 48.57 & 71 & 42.26 \\
\hline Neutral & 20 & 35.71 & 4 & 18.18 & 13 & 46.43 & 8 & 29.63 & 7 & 20.00 & 52 & 30.95 \\
\hline En desacuerdo & 1 & 1.79 & 0 & 0.00 & 0 & 0.00 & 0 & 0.00 & 2 & 5.71 & 3 & 1.79 \\
\hline $\begin{array}{l}\text { Totalmente en } \\
\text { desacuerdo }\end{array}$ & 0 & 0.00 & 0 & 0.00 & 1 & 3.57 & 0 & 0.00 & 0 & 0.00 & 1 & 0.60 \\
\hline Total & 56 & 100.00 & 22 & 100.00 & 28 & 100.00 & 27 & 100.00 & 35 & 100.00 & 168 & 100.00 \\
\hline
\end{tabular}

$\mathrm{X} 2=20.355 \mathrm{GL}=16$ P valor $=0.205$ No existe diferencia.

Fuente: Cuestionario dirigido a estudiantes de la muestra.

La tabla 11 está referida al reconocimiento de las fortalezas y debilidades que promueve la aplicación Quizizz al realizar diversas actividades. Se distingue que el 52,38\% del total de 168 estudiantes, se muestran de acuerdo en relación a la ayuda vital que proporciona Quizizz frente a sus fortalezas y debilidades mientras que una minoría del $4.17 \%$ no consideran que esta herramienta les favorezca en tal aspecto.

Respecto a lo mencionado, gran parte de los estudiantes encuestados reconoce que la herramienta Quizziz es muy gratificante para indagar y reconocer las debilidades y fortalezas existentes, lo cual favorece a los estudiantes para su plan de mejora generando en ellos su autoaprendizaje y autonomía. No obstante, se pudo establecer la existencia de un limitado número de estudiantes que no reconocen el valor significativo de Quizizz; lo cual podría incitar una nueva investigación.

\section{DISCUSIÓN DE RESULTADOS}

Con base al objetivo general de la presente investigación que fue conocer las apreciaciones del conectivismo a través de la herramienta Quizizz en tiempos de pandemia en los estudiantes de la carrera profesional de Idioma Extranjero, se puede discutir los siguientes ejes:

En la primera dimensión, se aclara que las TIC impactan en el aprendizaje en los tiempos de ahora, ya que estas están cada vez en constante avances (Meng et al., 2019) y acuerdo a los resultados de la presente investigación, ellas son útiles para el aprendizaje; motivo por el que los docentes están cada vez más inmersos en la tecnología, para su posterior incorporación en el aprendizaje (De Pablos 2012). La aplicación Quizizz acorde a los hallazgos, en donde presenta similitudes con el trabajo de Laura et al. (2021); el docente mediante la aplicación de dicha plataforma logra que los estudiantes participen, esto a que la herramienta permite que se incremente la atención de los estudiantes, por ende, su interés en la clase y autonomía en su aprendizaje. 
En la dimensión procedimental, es importante reconocer que el uso de las herramientas tecnológicas sirve para generar aprendizaje significativo en clase. En el presente trabajo se ve claramente que la muestra está de acuerdo con lo dicho con anterioridad; ya que los estudiantes viven en la era digital, por lo cual el docente tiene que usar herramientas digitales para promover la enseñanza (Mendoza, 2020). Por ello, en tiempos de pandemia, la enseñanza debe estar vinculada con los medios tecnológicos para que así pueda haber aprehensión de aprendizajes. En concordancia con la investigación de Ramírez et al. (2020) se encontró que los estudiantes están de acuerdo con el hecho de que el profesor guía a los estudiantes con el manejo de dicha herramienta; ya que en un inicio puede ser nueva para algunos estudiantes, sin un asesoramiento adecuado, esta herramienta en vez de ser útil se convertiría en una frustración para los estudiantes, ya que no saben su funcionamiento.

En la actitud de los estudiantes frente a esta herramienta, el 39.2\% lo encuentra emocionante, motivador y divertido, esto indica que los estudiantes si presentan una actitud positiva hacia la plataforma y un 35.3\% aseguran que la aplicación Quizizz cumple sus propósitos, dichos resultados se reafirman en el trabajo de Amalia (2020), puesto que la aplicación permite que los estudiantes vean que errores han cometido y reflexiones acerca de ello. Es importante en el proceso de enseñanza aprendizaje la retroalimentación, porque lo que se quiere es que el estudiante aprenda para la vida, y no solo para el examen.

En palabras de Ávila (2001), una limitación reside en el sentido de complejo interviniente que pausa o dificulta una investigación, particularmente un aspecto del problema es generado por alguna razón. Lo dicho hasta aquí supone que toda limitación debe ser justificada por una buena causa o motivo.

En el siguiente punto, describiremos algunos de los aspectos limitantes que intervinieron durante la ejecución del presente estudio. Por un lado, se observó un reducido número de estudiantes con dificultades para resolver el formulario virtual. Esto se debe a la baja señal de conexión a internet vivenciada en algunos hogares.

$\mathrm{Y}$, finalmente, en la parte de los resultados, aunque algunos de los datos recopilados tenían poca relevancia estadística, no se omitió ninguno de ellos. Sin embargo, se destacaron los más positivos como negativos, con la finalidad de precisar el impacto de la herramienta Quizizz en cada una de las interrogantes.

Los sistemas virtuales en los que se vio inmerso el ámbito educativo han generado popularidad a diversas herramientas educativas. De manera particular, la plataforma Quizizz ya que gratificantemente logró conectar con el aprendizaje de los estudiantes.

En este sentido, el presente estudio en referencia a la herramienta Quizizz invita a investigadores a actualizar y ampliar esta investigación para las futuras generaciones. Asimismo, innovar las metodologías empleadas por docentes, impulsarlos a indagar y conocer más sobre la herramienta presentada o alguna otra plataforma educativa que podría encajar con su metodología.

\section{CONCLUSIONES}

En primer lugar, con los resultados obtenidos, se concluye que los estudiantes consideran significante las herramientas tecnológicas para lograr un aprendizaje óptimo, 
puesto que la tecnología ya es parte de ellos, y que con la ayuda del docente pueden lograr una clase dinámica, donde los estudiantes pueden participar activamente.

De este modo, los estudiantes en su mayoría encuentran factible para lograr el aprendizaje significativo, pero que para que esto se dé; el docente tiene la gran función de ser el primero en aprender el uso de la herramienta, para posterior guiar a los estudiantes para que puedan tener idea de la plataforma web en la que están trabajando, esta herramienta es poderosa porque el hecho de que en si la aplicación sea dinámica, te permite que el aprendizaje sea más fácil.

Por último, los estudiantes sienten que la aplicación cumple con lo que se necesitan para estar motivados en la clase, así como el hecho de poder reconocer los errores que se cometen de manera personal, para su posterior mejora y reflexión, la retroalimentación es fundamental que los estudiantes interioricen ello para que la aplicación cumpla su propósito.

\section{ORCID}

Hillary Eddy Robles Gonzales: Universidad Nacional Jorge Basadre Grohmann, Tacna, Perú

Ruth Xiomara Salamanca Chaparro: Universidad Nacional Jorge Basadre Grohmann, Tacna, Perú (D)

Kevin Mario Laura De La Cruz: Universidad Nacional Jorge Basadre Grohmann, Tacna, Perú

\section{FUENTE DE FINANCIAMIENTO}

El estudio fue autofinanciado.

\section{CONFLICTOS DE INTERÉS}

Los autores expresan que no existen conflictos de interés.

\section{AGRADECIMIENTO}

No aplica.

\section{PROCESO DE REVISIÓN}

Este estudio ha sido revisado por pares externos en modalidad de doble ciego.

\section{DECLARACIÓN DE DISPONIBILIDAD DE DATOS}

No aplica.

\section{REFERENCIAS BIBLIOGRÁFICAS}

Amalia, D. F. (2020). Quizizz Website as an Online Assessment for English Teaching and Learning: Students' Perspectives. Jo-ELT (Journal of English Language Teaching) Fakultas Pendidikan Bahasa \& Seni Prodi Pendidikan Bahasa Inggris IKIP, 7(1). https://doi.org/10.33394/jo-elt.v7i1.2638

Basuki, Y., \& Hidayati, Y. (2019). Kahoot! or Quizizz: the Students' Perspectives. https://doi.org/10.4108/eai.27-4-2019.2285331 
Coll, C., Majós, M. T. M., \& Goñi, J. O. (2008). Análisis de los usos reales de las TIC en contextos educativos formales: Una aproximación socio-cultural. Revista Electronica de Investigacion Educativa, 10(1).

Cueva Delgado, J. L., García Chávez, A., \& Martínez Molina, O. A. (2019). El conectivismo y las TIC: Un paradigma que impacta el proceso enseñanza aprendizaje. Revista Scientific, 4(14). https://doi.org/10.29394/scientific.issn. 25422987.2019.4.14.10.205-227

De Pablos Pons, J. (2012). EL CAMBIO METODOLÓGICO EN EL ESPACIO EUROPEO DE EDUCACIÓN SUPERIOR Y EL PAPEL DE LAS TECNOLOGÍAS DE LA INFORMACIÓN Y LA COMUNICACIÓN. RIED. Revista Iberoamericana de Educación a Distancia, 10(2). https://doi.org/10.5944/ ried.2.10.992

Downes, S. (2007). What Connectivism Is. In Blog.

Gómez Reyes, F. M. (2020). Aplicaciones como medio e instrumento de evaluación: implementación de quizziz y kahoot en las evaluaciones de los estudiantes de contaduría pública. https://doi.org/10.4995/inred2020.2020.11972

Hernández Sampieri, R. Fernández Collao, C. (2016). Libro Metodología de la investigación SAMPIERI. In Libro Metodología de la investigación SAMPIERI.

Hernandez Sampieri, R., Fernandez Collado, C., \& Baptista Lucio, M. del P. (2010). Hernandez R, Fernández C, Baptista M. Metodología de la investigación. 5ta Ed.México: McGraw Hill; 2010. Metodología de La Investigación.

Laura, K., Morales, K., Clavitea, M., \& Aza, P. (2021). Aplicación Quizizz y comprensión de textos en inglés con el contenido de la plataforma educativa "Aprendo en Casa." Revista Innova Educación, 3(1). https://doi.org/10.35622/j.rie.2021.01.007

Mac Namara, D., \& Murphy, L. (2017). Online versus offline perspectives on gamified learning. CEUR Workshop Proceedings, 1857.

Mendoza Batista, A. M. (2020). Quizlet, Quizizz, Kahoot \& Lyricstraining: aprendizaje lúdico digital de una segunda lengua. Revista Lengua y Cultura, 1(2). https://doi. org/10.29057/lc.v1i2.5438

Meng, C. K., Nasir, J. S. B. M., Ming, T. M., \& Choo, K. A. (2019). A Gamified Classroom with Technical and Vocational Education and Training (TVET) Students using Quizziz. International Journal of Education, Islamic Studies and Social Sciences Research (IJEISR), 4(1).

Ovalles Pabón, L. C. (2014). Conectivismo, ¿un nuevo paradigma en la educación actual? - Dialnet. Fundación de Estudios Superiores Comfanorte FESC, 4.

Purba, L. S. L. (2020). The effectiveness of the quizizz interactive quiz media as an online learning evaluation of physics chemistry 1 to improve student learning outcomes. Journal of Physics: Conference Series, 1567(2). https://doi. org/10.1088/17426596/1567/2/022039 
Quispe, B. M., Cuadros Paz, L., Cornelio Fernández Gambarini, W., Palomino, Y. A., Addison, A., \& Quispihuanca, C. (2019). Análisis de las herramientas de gamificación online Kahoot y Quizizz en el proceso de retroalimentacion de aprendizajes de los estudiantes. Referencia Pedagógica, 7(2), 342-343. https://rrp.cujae.edu.cu/index.php/rrp/article/view/193

Ramírez-Ramírez, L. N., Caludio-Martínez, C., \& Ramírez-Arias, V. (2020). Usabilidad de las TIC en la Enseñanza Secundaria: Investigación-Acción con Docentes y Estudiantes de México. Revista Hallazgos 21, 5(1).

Siemens, G. (2005). Connectivism: A learning theory for the digital age. International Journal of Instructional Technology and Distance Learning, 2(1).

Soto, M. J. T., \& Larenas, C. H. D. (2021). QUIZIZZ Y SMARTPHONES: ESTRATEGIA DE CALENTAMIENTO PARA MEJORAR LA PARTICIPACIÓN EN CLASES DE LOS ESTUDIANTES UNIVERSITARIOS. Chakiñan, Revista de Ciencias ....

Valero Palomino, F. R., \& Bullón Solís, O. (2021). La virtualización, una alternativa viable en las instituciones educativas. PURIQ, 3(1). https://doi.org/10.37073/ puriq.3.1.139

Zhao, F. (2019). Using quizizz to integrate fun multiplayer activity in the accounting classroom. International Journal of Higher Education, 8(1). https://doi.org/10.5430/ijhe.v8n1p37

\section{CITAR COMO:}

Robles Gonzales, H. E., Salamanca Chaparro, R. X., \& Laura De La Cruz, K. M. (2022). Quizizz y su aplicación en el aprendizaje de los estudiantes de la carrera profesional de idioma extranjero. Puriq, 4, e239. https://doi.org/10.37073/puriq.4.1.239 